\title{
Absorption of isoflavones in humans: effects of food matrix and processing
}

Sonia de Pascual-Teresa ${ }^{1 *}$, Jesper Hallund ${ }^{2}$, Duncan Talbot ${ }^{3}$, Joyce Schroot $^{4}$, Christine M. Williams ${ }^{5}$, Susanne Bugel ${ }^{2}$ and Aedin Cassidy ${ }^{6}$

${ }^{1}$ CSIC. Instituto del Frio, Jose Antonio Novais, 10. 28040 Madrid. Spain

${ }^{2}$ The Royal Veterinary and Agricultural University. Department of Human Nutrition and

Centre for Advanced Food Studies, The Royal Veterinary and Agricultural University,

Rolighedsvej 30, DK-1958 Frederiksberg, Denmark

${ }^{3}$ Unilever Research. Biosciences Division, Unilever Research, Colworth House,

Sharnbrook, Bedfordshire MK 44 ILQ, UK

${ }^{4}$ Wageningen UR, Agrotechnology and Food Innovations B.V., Bornsesteeg 59, 6708

PD Wageningen, Holland

${ }^{5}$ The University of Reading. Hugh Sinclair Human Nutrition Unit, School of Food

Biosciences, Whiteknights, Reading RG6 6AP, UK

${ }^{6}$ University of East Anglia. School of Medicine, Norwich NR4 7TJ

*Corresponding author: Sonia de Pascual-Teresa

Telephone: +34 915492300, Fax number: +34 915493627

e-mail address: soniapt@if.csic.es

This work was supported by the EU-grant Isoheart QLK1-CT-2001-00221.

Running title: food matrix effect on isoflavones absorption

Keywords: Isoflavones; genistein; daidzein; food processing; bioavailability 


\begin{abstract}
If soy isoflavones are to be effective in preventing or treating a range of diseases, they must be bioavailable, and thus understanding factors which may alter their bioavailability needs to be elucidated. However to date there is little information on whether the pharmacokinetic profile following ingestion of a defined dose is influenced by the food matrix in which the isoflavone is given or by the processing method used. Three different foods (cookie, chocolate bar and juice) were prepared and their contents on isoflavones was determined. We compared the urinary and serum concentrations of daidzein, genistein and equol following the consumption of three different foods each of which contained $50 \mathrm{mg}$ of isoflavones.

After the technological processing of the different test foods differences in aglycone levels were observed. The plasma levels of the isoflavone precursor daidzein were not altered by food matrix. Urinary daidzein recovery was similar for all three foods ingested with total urinary output $33-34 \%$ of ingested dose. Peak genistein concentrations were attained in serum earlier following consumption of a liquid matrix than a solid matrix although there was a lower total urinary recovery of genistein following ingestion of juice than that of the other two foods.
\end{abstract}




\section{INTRODUCTION}

Although dietary isoflavones, which are present in soybeans, have gained significant attention in relation to their risk:benefit profile for human health [1], there are still no clear guidelines on safe and efficacious doses. What is critical to the biological efficacy and safety of these compounds is a thorough understanding of their bioavailability from different soya foods.

Following ingestion, the isoflavone glucosides are hydrolysed by intestinal $\beta$ glucosidases to produce aglycones in the small intestine $[2,3]$ which are then either absorbed intact or further metabolised by intestinal microflora in the large intestine into other metabolites like equol and O-desmethylangolensin (ODMA) [4, 5]. In soyabeans, isoflavones are present in different chemical forms; aglycones, $\beta$-glucosides, malonyland acetyl-glucosides. However, although in soya foods the predominant form is the glucosides, food processing techniques can alter the ratio of glucosides present and fermentation processes can result in increases in the levels of aglycones present in commercially available soya products. The major isoflavones in soyabeans are daidzin and genisitin as glycosides and their corresponding aglycone forms, daidzein and genistein [6].

Several recent studies have investigated the potential relationship between isoflavones in the glucoside and aglycone form from both pure compounds and soy foods to assess their effects on absorption, distribution, metabolism and excretion in animal and human studies $[7,8,9]$. However, some data reported that isoflavone aglycones (present in fermented soya products) were absorbed more efficiently than isoflavone glycosides [10] while other data suggests that the resulting bioavailability of daidzein and gensitein was 
greater when the soy isoflavones were ingested as glucosides rather than aglycones [11] and in one study no different in the apparent bioavailability following consumption of aglycone or glucoside tablets [12]. The distinct disparity in these results may be explained by factors such as age, gender or the type of soy food or isoflavone preparation fed. However what is ultimately important is to understand if the levels attained in urine and serum are similar following consumption of a known dose of isoflavones but present in different food matrices.

Therefore in the current study, we have examined the effect of food matrix on the apparent bioavailability of isoflavones from soy foods. A group of postmenopausal women were studied on three separate occasions and were randomly assigned to consume three different food matrices which each containing $50 \mathrm{mg}$ of soy isoflavones.

\section{METHODS AND MATERIAL}

\section{Chemicals}

All chemicals used were of analytical grade. Special reagents were daidzein, daidzin, 6"O-acetyldaidzin, genistein, genistin, 6"-O-acetylgenistin, glycitein, glycitin and 6"-Oacetylglycitin (LC laboratories, Woburn, USA), pancreatine from porcine pancreas, bile extract from pork, pepsine from porcine stomach and trypsine from bovine pancreas (Sigma) .

\section{Sample preparation}

$455 \mathrm{mg}$ of an isoflavone rich extract with a total isoflavone content of $11 \mathrm{wt} \%$ (SoyLife Extra®, Acatris B.V., Giessen, The Netherlands) was incorporated into three different food products; fruit juice (Döhler), chocolate bars (VSI) and cookies (A\&F). The criteria 
used for test food selection were: liquid-solid matrix effect, thechnological suitability as isoflavones carriers and consumers preferences within the group of study. Apple juice, orange juice or mandarin juice served as starting beverage compound together with sugar, citric cid, aroma and less than $0.1 \% \mathrm{CO}_{2}$. After addition of SoyLife Extra ${ }^{\circledR}$ the beverage was diluted with water to ready-to-drink strength produced the fruit juice. The resulting beverage was filled in bottles and pasteurized $\left(10 \mathrm{~min} 80^{\circ} \mathrm{C}\right)$. Chocolate bars were made by mixing SoyLife Extra ${ }^{\circ}$ and the syrup components at maximum $55^{\circ} \mathrm{C}$. It was casted in a flat mold, cooled, sliced into pieces and coated with chocolate. The cookies were made by mixing SoyLife Extra ${ }^{\circledR}$ with margarine, self-rising flour, wholemeal flour, sugar and spices and the baked for $15 \mathrm{~min}$ at $150^{\circ} \mathrm{C}$. Juice, cookies and bars contained respectively (g per serving): protein, 0, 2 and 18; fat, 0, 8, and 6; carbohydrates, 40, 20 and 22. Placebo foods were prepared in the same way as the isoflavone enriched foods.

\section{Isoflavone content and composition of the products}

A representative sample $(2 \mathrm{~mL}$ or $2 \mathrm{~g}$ ) was taken from all of these three products $(\mathrm{n}=3$ samples of each food) and the isoflavone content was determined by extraction and HPLC. Samples were extracted by stirring with $20 \mathrm{~mL} 50 \%$ acetonitrile/50\% MilliQ water for 2 hours at room temperature. The obtained solution was filtrated (Spartan 30 $0.45 \mu \mathrm{m}$ RC filter units) and if necessary diluted with extraction medium. The sample was injected on a Waters 2690 separations module with a column oven and a Waters 960 PDA detector attached to it. Separation was achieved using a Symmetry C18 column (250 x $4.6 \mathrm{~mm}, 5 \mu \mathrm{m})$. The chromatographic conditions were as follows: flow 1.5 $\mathrm{mL} / \mathrm{min}$; volume of injection $10 \mu \mathrm{L}$; column temperature $40{ }^{\circ} \mathrm{C}$; and solvents, $\mathrm{A}, 10 \mathrm{mM}$ ammonium formate in MilliQ water ( $\mathrm{pH} 2.8$ with formic acid) and $\mathrm{B}$, gradient grade 
acetonitrile. The gradient consisted of: 15\% of B isocratic for 5 min, 15-29\% of B for 31 min and $29-35 \%$ of B in 4 min. Detection was carried out in a diode array detector. PDA data between 225 and $300 \mathrm{~nm}$ were collected and a signal of $260 \mathrm{~nm}$ was extracted for integration. Peaks were identified on the basis of their retention time and UV spectrum against previously recorded standards. Daidzein, daidzin, 6"-O-acetyldaidzin, genistein, genistin, 6"-O-acetylgenistin, glycitein, glycitin and 6"-O-acetylglycitin were quantified and the results expressed as total isoflavone content and percentage of aglycones, glycosides and acetyl isoflavones.

\section{In vitro digestion of the isoflavone enhanced foods}

For the digestion $2 \mathrm{~g}$ food were suspended in $20 \mathrm{~mL}$ MilliQ water. $8 \mathrm{~mL}$ stomach fluid (pH 2.5) was added and the mixture kept at $37^{\circ} \mathrm{C}$ in a shaking water-bath for $1 \mathrm{~h}$. Immediately after, $10 \mathrm{~mL}$ intestine juice, $0.4 \mathrm{ml}$ trypsin solution and $0.4 \mathrm{ml}$ buffer solution were added and the $\mathrm{pH}$ was adjusted to 6.5 using $1 \mathrm{M} \mathrm{NaHCO}_{3}$. The mixture was kept at $37{ }^{\circ} \mathrm{C}$ in a shaking water bath for another $2 \mathrm{~h}$. After the digestion was finished a 1:1 dilution was made by adding $100 \%$ acetonitrile and the resulting solution injected in the HPLC for analysis.

The stomach fluid contained $0.07 \mathrm{mg}$ pepsin per $\mathrm{ml}$ stomach electrolyte. Stomach electrolyte solution contained $53 \mathrm{mM} \mathrm{NaCl}, 1 \mathrm{mM} \mathrm{CaCl}_{2}, 14.8 \mathrm{mM} \mathrm{KCl}$ and $5.7 \mathrm{mM}$ $\mathrm{Na}_{2} \mathrm{CO}_{3}$ and was adjusted to $\mathrm{pH} 2.5$ using $2 \mathrm{M} \mathrm{HCl}$. The intestine juice was made in two consecutive steps. First $25 \mathrm{~g}$ intestine electrolyte was mixed with $7 \mathrm{~g}$ pancreatine and 73 gr MilliQ water. This solution was mixed for 10 minutes and subsequently centrifuged for 20 minutes at $4{ }^{\circ} \mathrm{C}$ and $9800 \mathrm{~g}$. In the second step $0.475 \mathrm{~g}$ bile was added to $25 \mathrm{~mL}$ 
supernatant. The intestine electrolyte contained $21 \mathrm{mM} \mathrm{NaCl}, 2 \mathrm{mM} \mathrm{KCl}$ and $0.5 \mathrm{mM}$

$\mathrm{CaCl}_{2}$.

\section{Recruitment and screening of subjects}

\section{Subjects}

The single oral bolus dose food studies were conducted on postmenopausal women recruited from the staff and adult population from within and around the University of Reading, United Kingdom (UK) and The Royal Veterinary and Agricultural University, Frederiksberg, Denmark (DK). 12 postmenopausal women were enrolled to participate in these studies, 6 in DK and 6 in UK All individuals were healthy, had normal liver and kidney function accessed by serum aspartate amine transpherase (ASAT), alanine amino transpherase (ALAT) and creatinine, reported no use of medications (including antibiotic use within the preceding 3 months), no HRT users, did not consume soy-rich foods on a regular basis and reported no menstrual bleeds for at least two years prior to the commencement of the study. The study design and protocol were approved by the University of Reading and the Municipal Ethical Committee of Copenhagen and Frederiksber and informed consent was obtained from each subject following a detailed explanation of the study procedure.

\section{Design of study}

Subjects were asked to refrain from eating foods containing soy products for at least one month prior to the start of the study and for the duration of the study. Following an overnight fast, each individual arrived at the Reading and Frederiksberg research centres ( $n=6$ per study site) and following a standardised breakfast, were randomly assigned 
(using a multiple cross-over design) to receive one of the three isoflavone-rich foods on three separate occasions. Each oral dosing was separated by a minimum of one week. All subjects received the three different food items; cookies, chocolate bar, and juice (with an average content of $53.0 \pm 3.9 \mathrm{mg}$ isoflavones in which aglycones represented $2.0 \%$ and the ratio ratio of daidzein and its glucosides over genistein and its glucosides was $5.0 \pm$ $0.1)$ together with the standardised breakfast at each visit.

A baseline $10 \mathrm{ml}$ blood sample was collected prior to soy food ingestion. After the ingestion of the isoflavone-rich food over a period of 20 minutes (under supervision to ensure complete intake), further blood samples were obtained at 4, 6, 8, 12, 24, 48hrs after dosing. Blood was obtained by venepuncture .2x $5 \mathrm{ml}$ Blood samples were centrifuged at $1600 \mathrm{~g}$ for $10 \mathrm{~min}$ at $4^{\circ} \mathrm{C}$ and aliquots of serum $(2 \times 0.5 \mathrm{ml})$ stored at $-20^{\circ} \mathrm{C}$ until further analysis.

$24 \mathrm{hr}$ urine collections were made over the 48 hour study period. 4 x $25 \mathrm{ml}$ aliquots from each $24 \mathrm{hr}$ collection were stored at $-20^{\circ} \mathrm{C}$ until further analysis

\section{Serum and urinary isoflavone analysis}

Daidzein, Genistein and Equol concentrations were determined in serum and urine by Time-Resolved Fluoroimmunoassay (TR-FIA). Samples were analysed for daidzein using a previously published TR-FIA [13]. Based on this established format, similar TRFIA assays for genistein and equol, using monoclonal antibodies prepared by the Unilever Colworth monoclonal group, were developed. Commercial assays were not available at the time. 
$25 \mathrm{~mL}$ urine was pre-incubated in an anti-mouse plate (Perkin Elmer Product Code (PEPC) AAAND-00033) with 100mL Europium labeled daidzein, genistein or equol tracer, diluted in DELFIA assay buffer (PEPC 13803185). For the quantitative analysis of daidzein and equol the urine was pre-treated with $400 \mathrm{U} / \mathrm{mL}$ of B-Glucuronidase (Sigma G7396) by incorporation of the enzyme in the tracer diluent. 100mL monoclonal antibody, diluted in assay buffer, was then added and the plate incubated for a further hour (Daidzein: Clone 4E4 Fortune Kohen, Weizmann Institute1100ng/ml. Genistein: inhouse clone $6547.3,65 \mathrm{ng} / \mathrm{ml}$ and equol in-house clone $6588.1,20 \mathrm{ng} / \mathrm{ml}$ ). Plates were then washed six times with DELFIA wash buffer (PEPC 1244-114) and 200mL enhancement solution (PEPC 1244-105) added. Following shaking for a further 5 minutes quantitative data was then attained by reading on an AutoDelfia in the case of automated analysis or on a Victor2 Multilabel Counter for assays performed manually.

Daidzein analysis was performed on non-extracted serum samples according to the method outlined for urinary analysis, whilst for genistein and equol analysis prior sample extraction was performed. The extraction method was based on the one described by Labmaster (Wang 20003 and Brouwers 20034) for use with blood samples to be assayed in their commercial daidzein, genistein and equol TR-FIA kits. Glucuronides and sulphates were hydrolysed by adding $200 \mathrm{~mL} 0.1 \mathrm{M}$ acetate buffer $\mathrm{pH} 5$ containing $0.2 \mathrm{U} / \mathrm{mL}$ of B-glucuronidase (Boehringer Mannheim Cat. No. 1585665) and 2U/mL of sulphatase (Sigma Prod. Code S9626) to 200mL serum. After mixing, the samples were incubated at $370 \mathrm{C}$ overnight. Thereafter equol and genistein was extracted with $1.5 \mathrm{~mL}$ diethyl ether. The water phase was frozen in a dry ice - ethanol mixture and the ether phase transferred into a glass tube. After thawing the water phase was re-extracted with 
ether and the ether phases were combined and evaporated to dryness in a $+450 \mathrm{C}$ water bath. 200mL DELFIA assay buffer was then added to each tube and they were vortexed to reconstitute the extracted sample prior to DELFIA analysis.

The genistein assay on extracted serum used an alternative in-house antibody 6606.1 with a more appropriate sensitivity and specificity profile for blood analysis (Genistein: inhouse clone 6606.1, $200 \mathrm{ng} / \mathrm{ml})$. Otherwise assay conditions were as described for urine analysis.

\section{Statistical analyses and Pharmacokinetic parameters}

Statistical analysis was performed by using the SPSS software package for Windows (version 11.5.1, SPSS, Richmond, CA). Values are reported as means \pm SEMs unless noted otherwise, and the significance level was set at $\alpha=0.05$. The area under the curve (AUC) from 0 to $24 \mathrm{~h}$ was calculated by using the trapezoidal rule and serum genistein, daidzein and equol measured at $0,4,6,8,12,24$, and $48 \mathrm{~h}$ after a single oral dose administered at time 0 . Maximum serum concentrations of the three isoflavones from 0 to $48 \mathrm{~h}$ post-dose were defined as $c_{\max }$. The time to maximum serum concentration $\left(t_{\max }\right)$ was defined as the time in hours at which $c_{\max }$ was reached. The elimination half-life for genistein, daidzein and equol was computed by using the following formula: $t_{1 / 2}=-$ $\ln (2) / \beta$, where $\beta$ is the slope of the linear regression of the $\ln$ of serum genistein, daidzein and equol concentrations 8,12, 24 and $48 \mathrm{~h}$ after isoflavones enriched foods. 


\section{RESULTS}

\section{Isoflavone contents in the foods}

In Figure 1 we show a chromatogram corresponding to one of the analysed foods as an example of the profile of isoflavones obtained. Daidzein, daidzin, 6"-O-acetyldaidzin, genistein, genistin, 6"-O-acetylgenistin, glycitein, glycitin and 6"-O-acetylglycitin where all present in all the analysed samples.

In the isoflavone enriched food materials less than $4 \%$ total aglycones were present (including glycitein) (Table 1). About 65 to $75 \%$ of the total isoflavone content was present as glucoside, the remaining part as acetylglucoside. The D/G ratio was $5.0 \pm 0.2$ (95\% CI) for the prepared foods. Isoflavones concentrations were not significantly different in the three tested products.

\section{In vitro digestion of the isoflavone enhanced foods}

After in vitro digestion the cookies differed significantly from the other two product types in \% recovery of total isoflavones (see Table 2). No significant difference in \% recovery was observed between bars and juice. Significant differences where also observed in the case of the cookies between the ratio in the original food and after in vitro digestion. Proportionally, in this model system, a higher amount of genistein was absorbed compared with daidzein.

\section{In vivo absorption of isoflavones from the isoflavone enhanced foods}

Twelve healthy volunteers (six in UK and six in DK) were recruited and completed the three phases of the study. Pre-study screening data are given in Table 3.

Volunteers were fed a food containing the $50 \mathrm{mg}$ of isoflavones, as either, a cookie, chocolate bar or juice. Based on the $50 \mathrm{mg}$ isoflavones, the subjects each consumed: 6.7 
mg genistein and $33.8 \mathrm{mg}$ daidzein, both predominantly in the glycoside form (aglycones $<4.4 \%$ in every case)

\section{Serum levels}

Serum genistein was $0.9 \pm 5.39 \mathrm{ng} / \mathrm{ml}, 0.9 \pm 4.62 \mathrm{ng} / \mathrm{ml}$ and $2.0 \pm 11.89 \mathrm{ng} / \mathrm{ml}$ prior to ingestion of cookies, bars and juice respectively. Prior to isoflavone administration, serum daidzein was $0.1 \pm 1.55 \mathrm{ng} / \mathrm{ml}, 0.2 \pm 1.12 \mathrm{ng} / \mathrm{ml}$ and $0.1 \pm 1.37 \mathrm{ng} / \mathrm{ml}$, while baseline serum equol concentrations were $0.6 \pm 0.57 \mathrm{ng} / \mathrm{ml}, 0.5 \pm 0.26 \mathrm{ng} / \mathrm{ml}$ and $0.4 \pm$ $0.23 \mathrm{ng} / \mathrm{ml}$ respectively. Changes in serum total genistein, daidzein and equol concentrations during the $48 \mathrm{~h}$ after ingestion of the meal varied substantially between the individuals.

After consumption of the test foods containing isoflavones, serum genistein concentrations increased to $160.9 \pm 74.88 \mathrm{ng} / \mathrm{ml}, 157.5 \pm 61.74 \mathrm{ng} / \mathrm{ml}$ and $129.1 \pm 86.22$ $\mathrm{ng} / \mathrm{ml}$ in the cookies, bars and juice respectively (Figure 2a). Peak genistein concentrations were reached at time $8 \mathrm{~h}$ for cookies and bars and at time $6 \mathrm{~h}$ following consumption of the juice.

Serum daidzein concentrations increased to a maximum concentration of $116.8 \pm 28.67$ $\mathrm{ng} / \mathrm{ml}, 127.2 \pm 20.36 \mathrm{ng} / \mathrm{ml}$ and $112.1 \pm 30.37 \mathrm{ng} / \mathrm{ml}$ following ingestion of the cookies, bars and juice respectively (Figure 2b). Peak daidzein concentration was attained at time $8 \mathrm{~h}$ for all the tested foods.

Negligible increases in serum equol concentrations were observed in this study group; 1.2 $\pm 0.41 \mathrm{ng} / \mathrm{ml}, 1.1 \pm 0.40 \mathrm{ng} / \mathrm{ml}$ and $1.2 \pm 0.52 \mathrm{ng} / \mathrm{ml}$ following ingestion of the cookies, bars and juice respectively (Figure 2c). However, peak equol concentrations were reached at $12 \mathrm{~h}$ for all the tested foods. 
Forty-eight hours after ingestion of the isoflavone-enriched foods, mean serum genistein concentrations had dropped to $8.5 \pm 16.49 \mathrm{ng} / \mathrm{ml}, 3.8 \pm 6.51 \mathrm{ng} / \mathrm{ml}$ and $2.5 \pm 6.93 \mathrm{ng} / \mathrm{ml}$ in the three study phases. Serum daidzein was $0.7 \pm 2.14 \mathrm{ng} / \mathrm{ml}, 0.4 \pm 2.75 \mathrm{ng} / \mathrm{ml}$ and 0.8 $\pm 1.84 \mathrm{ng} / \mathrm{ml} 48 \mathrm{~h}$ after ingestion of cookies, bars and juice respectively. AUC, $c_{\max }, t_{\max }$ and $t_{1 / 2}$ values were calculated for serum total genistein, daidzein and equol after ingestion of the isoflavone enriched cookies, bars and juice (Table 4).

The $48 \mathrm{~h}$ AUCs for genistein, daidzein and equol after ingestion of either cookies, bar or juice were not significantly different and averaged $9.28 \pm 0.39,7.49 \pm 1.02$ and $0.16 \pm$ 0.01 respectively. Ingestion of cookies, bar or juice resulted in $t_{\max }, c_{\max }$, and $t_{1 / 2}$ values that were not significantly different.

\section{Urinary levels}

$50 \%$ of the ingested genistein was eliminated in urine in the first 24 hours and a further $11 \%$ on the second study day after consumption of the juice. In the case of cookies and bars 56\% and 55\% were eliminated after 24 hours respectively and a further $12 \%$ and $11 \%$ on the second day (Figure 3a).

For daidzein $31 \%, 30 \%$ and $29 \%$ of the dose was excreted in urine in the first 24 hours after ingestion of cookies, bars and juice respectively and a further $4 \%$ on the second day (Figure 3b).

Approximately half of the excreted equol was determined in urine in the first 24 hours and the rest on the second day (Figure 3c).

\section{DISCUSSION}

Isoflavones may be important in the prevention and or treatment of a range of chronic diseases and data from in vitro model systems, animal studies and intervention trials in 
humans attest to the biological activity of these compounds and their potential importance to human health [1]. Although rates of hormone dependent diseases are lower in populations who consume relatively high intakes of soy isoflavones, if soy isoflavones are to be effective in preventing or treating a range of diseases, they must be bioavailable, and thus understanding factors which may alter their bioavailability needs to be elucidated.

It is well established that soy isoflavones are readily absorbed from the gastrointestinal tract and reach peak concentrations within a few hours of ingestion [11]. A small number of studies have evaluated the levels of soy isoflavones attained in serum or urine but many have either focused on a single food $[7,8,9]$ or studied specific purified aglycones or glycosides $[10,11]$. There is little information, however, as to whether the appearance and disappearance of isoflavones in urine and serum is influenced by the food matrix in which the isoflavones are contained. The aim of this study was to determine the effect of food matrix on the levels of isoflavones attained in serum and urine in a group of healthy postmenopausal women given an oral dose of three different foods containing a defined level of isoflavones $(50 \mathrm{mg})$ on three separate occasions.

The studies were preformed according to a classic single oral bolus dose pharmacokinetic design, using a level of intake which was reflective of habitual intake in Asia and are complementary to previously published studies which used stable isotopes of the pure compounds or soy foods $[9,14]$. These data clearly demonstrate that humans absorb isoflavones from a range of different food matrices.

In previous studies, several investigators have use a liquid matrix, like soymilk [15], specific soy beverages [16] or soy-rich preparations suspended in liquids [7, 8]. In the 
present study, $t_{\max }$ following juice consumption was $7.8 \pm 1.8 \mathrm{~h}$ and $7.0 \pm 0.1 \mathrm{~h}$ for daidzein and genistein respectively. This is in agreement with other studies employing liquid food preparations where the mean $t_{\max }$ occurs between 6 and $9 \mathrm{~h}$ for both daidzein and genistein $[7,8,16,17]$.

When we compared the isoflavone concentrations after the technological processing of the different test foods we only found differences in aglycone levels. For the cookies, the level of aglycones, of both genistein and daidzein, were slightly higher ( $3.0 \pm 1.4)$ than those in the juice or chocolate bars $(1.5 \pm 2.5$ and $1.5 \pm 0.7$ respectively). However these differences did not seem to have any effect on the Cmax obtained after the ingestion of the three test foods.

With regards to the in vitro bioavalability assay, the results showed a very low recovery of isoflavones from the cookies. This result could be due to the high complexity of the matrix of the cookies, with a high content of sugars and proteins, which may make the extraction of isoflavones more difficult. However these results were not reflected in the human study and add weight to the difficulties in extrapolating results from in vitro experimentation to help understand the human absorption of bioactive compounds from food.

In agreement with previous studies, wide inter-individual variability in the urinary recovery of daidzein and genistein was observed $[18,19]$. In the current study $(\mathrm{n}=12)$, recovery of total daidzein and genistein was 38.8\% ( \pm 5.3$)$ of total isoflavones ingested with daidzein and genistein recoveries ranging from $32-35 \%$ and $61-70 \%$. Following soy food consumption, it is well established that urinary daidzein concentrations are consistently higher than urinary genistein levels $[7,8,9,15]$. Given the profile of the soy 
isoflavone extract (41.8 $\mathrm{mg}$ daidzein, $8.2 \mathrm{mg}$ genistein) added to our three food matrices, it was thus not surprising that daidzein levels in urine were consistently higher than urinary genistein levels. However in the present study the percentage of genistein eliminated in urine was higher than that of daidzein. This is possibly due to the fact that in our study the relative proportions of daidzein:genistein in the food were 5:1 while in most of the previous studies the proportion is approximately 1:1.

The excretion profiles of daidzein and genistein over the $48 \mathrm{hrs}$ following ingestion of the soy foods are similar to that which we previously observed [9] with the majority appearing in the urine within the first $24 \mathrm{~h}$ following the ingestion of the soy food. This rapid appearance of these compounds in urine can be explained by the short serum halflife and fast serum clearance rates of both daidzein and genistein (Table 4). Other investigations of the urinary excretion patterns of these compounds following acute ingestion have also reported low excretion rates $24 \mathrm{~h}$ post ingestion $[7,8,9]$. The current study aimed to evaluate the potential impact that food matrix may have on urinary isoflavone excretion following the consumption of a single portion of three foods containing a known dose of soy isoflavones in the same group of subjects. There was a lower total urinary recovery of genistein following ingestion of juice $(61 \%)$ than that of the other two foods (66\% and 70\% for bars and cookies respectively). Daidzein recovery remained similar across the three foods ingested with total urinary output $32-35 \%$ of ingested intake. These data suggest that the levels of isoflavones attained, particularly levels of genistein, may be altered depending on the food matrix consumed, but the current data did not reach statistical significance. It should be noted that for the given variance in this study for a study power of $80 \%$ a minimum of 48 volunters would be 
necessary in order to get significancy for a $13 \%$ difference in isoflavone excretion at a $\mathrm{P}<0.05$.

The metabolites of daidzein and genistein, formed in the intestine may account for some of the rest of the urinary isoflavones levels and various other metabolites have been identified in biological fluids $[20,21,22,23]$. In this study we focused on the metabolite of daidzein, equol, as a marker of intestinal metabolism. Equol has been the focus of much interest because it is more oestrogenic and a more potent antioxidant than daidzein $[24,25,26]$ suggesting that this isoflavone metabolite may hold the key to understanding the mechanism of action and effectiveness of soya in clinical studies examining the potential health benefits of soya isoflavones. It is well established that only $30 \%$ of any given population group studied can produce equol $[19,27,28,29]$ and an inability to produce equol may be related to an absence of appropriate enzymes in the intestinal microflora or absence of bacterial species capable of producing equol [20, 30]. Its formation is exclusively related to intestinal microflora as germ free rats do not excrete equol and the absence of equol from infant blood samples following soy infant formula ingestion add weight to the need for an active microflora for its formation [31]. Our studies in healthy adults using C13 daidzein and C13 gensitein show conclusively that equol is formed from daidzein and not genistein [9]. The question of whether we can enhance intestinal conversion of daidzein to equol is one of great interest $[17,20]$ and dietary carbohydrate has been indicated as a factor that may determine equol production $[1,19,20,29]$. Surprisingly in the current study none of the subjects were equol producers. 
This study examined the potential effect of food matrix on the levels of isoflavones attained in urine and serum. The levels of the isoflavone precursor, daidzein did not appear to be altered by food matrix and in this study none of the volunteers appeared capable of converting this precursor to its intestinal metabolite, equol. Peak genistein concentrations were attained in serum earlier following consumption of liquid matrix than a solid matrix however these differences were not statisticaly significatives. Further studies are required to determine the relative importance of food matrix to ensure safe and efficacious doses of these compounds can be delivered for potential health benefits. The pharmacokinetic data gathered from this study relate well to the limited information available from previous work. More specifically, $C_{\max }, t_{\max }, t_{1 / 2}$ and $\mathrm{AUC}_{(0-\mathrm{t})}$ were all within the range of values obtained from other studies using similar quantities of dietary isoflavones $[7,8,9,11,15]$. Accurate measurements of bioavailability would ideally compare the AUC after both oral and intravenous administration. However in any study examining bioavailability, the accuracy is dependent on taking sufficient blood samples during the elimination phase and continuing with sampling ideally to at least five half lives beyond the time that steady state levels are reached in blood. However only a few studies on bioavailability have taken this into consideration $[7,8,11,31,32]$ while several studies have only used a couple of time points to compute the pharmacokinetic $[10,33]$. These inconsistencies in study design and sampling may well account for any discrepancies in the available literature. 


\section{ACKNOWLEDGMENT}

We thank the women who took part in this study. We also thank Laura Barrios, at the Department of Operational Research and Applied Statistics, CSIC, for statistical advice. 


\section{REFERENCES}

[1] Setchell, K. D.; Cassidy, A. Dietary isoflavones: biological effects and relevance to human health. J. Nutr. 1999, 129, 758S-767S.

[2] Day, A. J.; DuPont, M. S.; Ridley, S.; Rhodes, M.; Rhodes, M. J.; Morgan, M.

R.; Williamson, G. Deglycosylation of flavonoid and isoflavonoid glycosides by human small intestine and liver beta-glucosidase activity. FEBS Lett. 1998, 436, 7175.

[3] McMahon, L. G.; Nakano, H.; Levy, M. D.; Gregory, J. F. Cytosolic pyridoxine-beta-D-glucoside hydrolase from porcine jejunal mucosa. Purification, properties, and comparison with broad specificity beta-glucosidase. J. Biol. Chem. 1997, 272, 32025-32033.

[4] Axelson, M.; Kirk, D. N.; Farrant, R. D.; Cooley, G.; Lawson, A. M., Setchell, K. D. R. The identification of the weak estrogen equol (7-hydroxy-3-(4'hydroxyphenyl)chroman] in human-urine. Biochem. J. 1982, 201, 353-357.

[5] Axelson, M.; Sjovall, J.; Gustafsson, B. E.; Setchell, K. D. R. Soya - a dietary source of the non-steroidal estrogen equol in man and animals. J. Endocrinol. 1984, 102, 49-56.

[6] Coward, L.; Barnes, N. C.; Setchell, K. D. R.; Barnes, S. Genistein, daidzein, and their beta-glycoside conjugates - antitumor isoflavones in soybean foods from american and asian diets. J. Agric. Food Chem. 1993, 41, 1961-1967.

[7] King, R. A., Bursill, D. B. Plasma and urinary kinetics of the isoflavones daidzein and genistein after a single soy meal in humans. Am. J. Clin. Nutr. 1998, 67, 867-872. 
[8] Watanabe, S.; Yamaguchi, M.; Sobue, T.; Takahashi, T.; Miura, T.; Arai, Y.; Mazur, W.; Wahala, K.; Adlercreutz, H. Pharmacokinetics of soybean isoflavones in plasma, urine and feces of men after ingestion of $60 \mathrm{~g}$ baked soybean powder (kinako). J. Nutr. 1998, 128, 1710-1715.

[9] Setchell, K. D.; Brown, N. M.; Desai, P. B.; Zimmer-Nechimias, L.; Wolfe, B.; Jakate, A. S.; Creutzinger, V.; Heubi, J. E. Bioavailability, disposition, and doseresponse effects of soy isoflavones when consumed by healthy women at physiologically typical dietary intakes. J. Nutr. 2003, 133, 1027-1035.

[10] Izumi, T.; Piskula, M. K. ; Osawa, S. ; Obata, A. ; Tobe, K. ; Saito, M. ;

Kataoka, S. ; Kubota, Y. ; Kikuchi, M. Soy isoflavone aglycones are absorbed faster and in higher amounts than their glucosides in humans. J. Nutr. 2000, 130, 16951699.

[11] Setchell, K. D.; Brown, N. M.; Desai, P.; Zimmer-Nechemias, L.; Wolfe, B. E.; Brashear, W. T.; Kirschner, A. S.; Cassidy, A.; Heubi, J. E. Bioavailability of pure isoflavones in healthy humans and analysis of commercial soy isoflavone supplements. J. Nutr. 2001, 131, 1362S-1375S.

[12] Zubik, L.; Meydani, M. Bioavailability of soybean isoflavones from aglycone and glucoside forms in American women. Am. J. Clin. Nutr. 2003, 77, 1459-1465. [13] Kohen, F.; Lichter, S.; Gayer, B.; DeBoever, J.; Lu, L. J. W. The measurement of the isoflavone daidzein by time resolved fluorescent immunoassay: a method for assessment of dietary soya exposure. J. Steroid Biochem. Mol. Biol. 1998, 64, $217-$ 222. 
[14] Faughnan, M. S.; Hawdon, A.; Ah-Singh, E-; Brown, J.; Millward, D. J.;

Cassidy, A. Urinary isoflavone kinetics: the effect of age, gender, food matrix and chemical composition. Br. J. Nutr. 2004, 91, 567-574.

[15] Zhang, Y.; Wang, G. J.; Song, T. T.; Murphy, P. A.; Hendrich, S. Urinary

disposition of the soybean isoflavones daidzein, genistein and glycitein differs among humans with moderate fecal isoflavone degradation activity. J. Nutr. 1999, 129, 957962

[16] Richelle, M.; Pridmore-Merten, S.; Bodenstab, S.; Enslen, M.; Offord, E. A. Hydrolysis of isoflavone glycosides to aglycones by beta-glycosidase does not alter plasma and urine isoflavone pharmacokinetics in postmenopausal women. J. Nutr. 2002, 132, 2587-2592.

[17] Setchell, K. D.; Brown, N. M; Lydeking-Olsen, E. The clinical importance of the metabolite equol-a clue to the effectiveness of soy and its isoflavones. J. Nutr. 2002, 132, 3577-3584.

[18] Karr, S. C.; Lampe, J. W.; Hutchins, A. M.; Slavin, J. L. Urinary isoflavonoid excretion in humans is dose dependent at low to moderate levels of soy-protein consumption. Am. J. Clin. Nutr. 1997, 66, 46-51.

[19] Rowland, I.; Wiseman, H.; Sanders, T.; Adlercreutz, H.; Bowey, E. Metabolism of oestrogens and phytoestrogens: role of the gut microflora. Biochem. Soc. Trans. 1999, 27, 304-308.

[20] Axelson, M.; Sjovall, J.; Gustafsson, B. E., Setchell, K. D. Soya-a dietary source of the non-steroidal oestrogen equol in man and animals. J. Endocrinol. 1984, 102, 49-56. 
[21] Coldham, N. G.; Howells, L. C.; Santi, A.; Montesissa, C.; Langlais, C.; King, L. J.; Macpherson, D. D.; Sauer, M. J. Biotransformation of genistein in the rat: elucidation of metabolite structure by product ion mass fragmentology. J. Steroid. Biochem. Mol. Biol. 1999, 70, 169-184.

[22] Joannou, G. E.; Kelly, G. E.; Reeder, A. Y.; Waring, M.; Nelson, C. A urinary profile study of dietary phytoestrogens. The identification and mode of metabolism of new isoflavonoids. J. Steroid. Biochem. Mol. Biol. 1995, 54, 167-184.

[23] Kelly, G. E.; Nelson, C.; Waring, M. A.; Joannou, G. E.; Reeder, A. Y. Metabolites of dietary (soya) isoflavones in human urine. Clin. Chim. Acta. 1993, $223,9-22$.

[24] Shutt, D. A.; Cox, R. I. Steroid and phyto-oestrogen binding to sheep uterine receptors in vitro. J. Endocrinol. 1972, 52, 299-310.

[25] Arora, A.; Nair, M. G.; Strasburg, G. M. Antioxidant activities of isoflavones and their biological metabolites in a liposomal system. Arch. Biochem. Biophys. 1998, $356,133-141$.

[26] Mitchell, J. H.; Gardner, P. T.; McPhail, D. B.; Morrice, P. C.; Collins, A. R.; Duthie, G. G. Antioxidant efficacy of phytoestrogens in chemical and biological model systems. Arch. Biochem. Biophys. 1998, 360, 142-148.

[27] Cassidy, A.; Bingham, S.; Setchell, K. D. Biological effects of a diet of soy protein rich in isoflavones on the menstrual cycle of premenopausal women. Am J Clin Nutr. 1994, 60, 333-340. 
[28] Cassidy, A.; Bingham, S.; Setchell, K. D. Biological effects of isoflavones in young women: importance of the chemical composition of soyabean products. $B r . J$. Nutr. 1995, 74, 587-601.

[29] Lampe, J. W.; Karr, S. C.; Hutchins, A. M.; Slavin, J. L. Urinary equol excretion with a soy challenge: influence of habitual diet. Proc. Soc. Exp. Biol. Med. 1998, 217, 335-339.

[30] Setchell, K. D.; Borriello, S. P.; Hulme, P.; Kirk, D. N.; Axelson, M. Nonsteroidal estrogens of dietary origin: possible roles in hormone-dependent disease. Am. J. Clin. Nutr. 1984, 40, 569-578.

[31] Setchell, K. D. ; Zimmer-Nechemias, L. ; Cai, J. ; Heubi, J. E. Exposure of infants to phyto-oestrogens from soy-based infant formula. Lancet. 1997, 350, 23-27. [32] Fanti, P.; Sawaya, B. P.; Custer, L. J.; Franke, A. A. Serum levels and metabolic clearance of the isoflavones genistein and daidzein in hemodialysis patients. J. Am. Soc. Nephrol. 1999, 10, 864-871.

[33] Xu, X.; Wang, H. J.; Murphy, P. A.; Cook, L.; Hendrich, S. Daidzein is a more bioavailable soymilk isoflavone than is genistein in adult women. J. Nutr. 1994, 124, $825-832$ 
Figure 1: HPLC chromatograms recorded at $260 \mathrm{~nm}$ corresponding to a isoflavone enriched cookie. Peak identification: 1, daidzin; 2, glycitin; 3, genistin; 4, 6"-Oacetyldaidzin; 5, 6"-O-acetylglycitin; 6, daidzein; 7, genistin; 8, 6"-O-acetylgenistin; 9, genistein.

Figure 2: serum appearance / disappearance curves following ingestion of a known dose of soy isoflavones present in different foods: a, genistein, b, daidzein and c, equol.

Figure 3: Urinary isoflavone excretion (a, genistein; b, daidzein; c, equol) following the ingestion of a known dose of isoflavones present in different food matrices. 